\title{
Esthetic management of multiple missing anterior teeth - A Case report
}

\author{
Dr. Lalit Kumar ${ }^{1}$, Dr. Hemant Batra ${ }^{2}$ \\ ${ }^{I}$ Senior Lecturer, Department of Prosthodontics Dr Harvansh Singh Judge Institute of Dental Sciences \& \\ Hospital, Panjab University, Chandigarh India \\ ${ }^{2}$ Professor and Head, Department Of Oral and Maxillofacial Surgery Dr. Harvansh Singh Judge Institute \\ of Dental Sciences and Hospital, Panjab University, Chandigarh India
}

\begin{abstract}
The aim of this case report was to present management of a case of multiple apical root resorptions due to orthodontic treatment in a 27-year-old adult female using implant supported crowns. External root resorption of the permanent teeth is a multi-factorial process. Well recognized causes of apical root resorption in permanent teeth include orthodontic therapy, trauma, periapical or periodontal inflammation, tumors, cysts, occlusal stress, impacted teeth, systemic conditions, endocrine imbalances and dietary habits. This case report presents esthetic management of missing central incisors using gingival porcelain for esthetics without using any augmentation procedure.
\end{abstract}

Keywords: Ankylos, Gingival Porcelain, Implant, Orthodontic Resorption,

\section{Introduction}

Tooth movement induced by orthodontic appliances is one of the most frequent therapeutic procedures in clinical dental practice. The search for esthetics and functionality, both oral and dental, demands orthodontic treatments, which are often associated with root resorptions that may, in extreme cases, lead to tooth loss, periodontal damage, or both. Currently, endosseous implants are used with established success in various clinical situations. A significant problem, however, is insufficient height or width of the alveolar bone at the implantation site. This may be caused by resorption of the alveolar bone after infection, extraction, or trauma, or may be the result of failed orthodontic treatment. Restoration of missing teeth with implants in the anterior maxilla while maintaining acceptable interdental papillae presents a major restorative challenge. Full coverage of the implant surface with bone is a prerequisite for reliable insertion of an endosseous implant. Augmentation of local defects of the alveolar ridge with bone grafts may offer a method to enable implantation in cases with insufficient bone volume.

Several surgical procedures to create sufficient bone volume have been developed, such as autogenous bone grafts, including onlay grafts $[8,9,14]$ and interpositional bone grafts,[6,7] guided bone regeneration $[1,3,4,5,11]$ and combinations of these procedures. The choice between ridge augmentation or the use of a membrane in conjunction with implantation depends on the extent and shape of the bone defect. Implants should have primary stability in a position appropriate for prosthodontic rehabilitation. If primary stability or appropriate positioning cannot be achieved, ridge augmentation is recommended before implantation.[12]

However sometimes patient do not agree for graft procedures or any other bone augmentation procedures. In clinical situations like this, the clinician has to rely on some other method for restoration of an implant supported crown in the anterior maxilla while maintaining the acceptable interdental papillae. The contours and color of missing teeth may be reproduced using a variety of materials and techniques. The success of an implant-supported restoration is often limited by the provider's ability to create gingival contours that harmonize with a patient's remaining oral structures. The presence or absence of the interdental papilla associated with multiple adjacent implants may be affected by the amount of alveolar bone loss prior to an implant placement, distance between adjacent implants, position of implants in relation to adjacent teeth, as well as the subgingival contours of the implant-supported restoration. In many instances, the loss of 2 or more maxillary anterior teeth results in flattening of edentulous segments and resorption of inter proximal bone crests and placement of implants may result in additional bone loss following bone remodeling and the establishment of biological widths.

Many surgical techniques have been developed attempting to regenerate interdental papillae. Unfortunately, no single technique offers consistent clinical success. Therefore, when using implants to replace multiple maxillary anterior teeth, optimal implant positioning should be achieved. When a diminished mesialdistal space exists, the clinician should consider placing implants with smaller diameters.[13] Alternatively, fewer implants with cantilevered fixed partial dentures may be considered. In each instance, the intent is to maximize the inter implant distance, minimize alveolar bone loss, and maintain acceptable soft tissue height for 
optimum esthetic results. This clinical report illustrates an approach to manage resorbed maxillary alveolar ridge associated with multiple implants.

Ridge mapping is a measurement procedure to ensure that the diameter of an endosseous screw implant does not exceed the dimensions of available bone. The long-term success of implants is a prime aim. To achieve this, it is essential for the initial evaluation of the dimensions of the resorbing alveolar process to be absolutely accurate. Ideally, an implant should be covered by at least $1 \mathrm{~mm}$ of bone on all sides. The major problem is estimating the thickness of bone, since the mucosal contour can mask the actual dimension of the alveolar ridge. Use of ridge mapping makes possible a reliable evaluation procedure other than CBCT or Denta scan at the initial stage of treatment planning.

\section{Case Report}

A 27 year old healthy female reported to department of prosthodontics with mobile maxillary central incisors and for dental replacement for maxillary central incisors (Fig.. 1) which were lost because of failed orthodontic treatment. Clinical examination revealed missing central incisor and radiological examination revealed the resorbed maxillary central and lateral incisor (Fig.. 2). Options of therapy considered were a removable appliance, a fixed partial denture of some type, or two implant restorations. Interim removable partial denture was fabricated in relation to maxillary central incisors. Ridge mapping was done to confirm the approximate labio lingual width of the edentulous area.

Following day of surgery prophylactic antibiotic (Augmentin $625 \mathrm{mg}$ t.i.d for 5 days) was given orally. Using 15 no. blade a supra crestal incision was given and mucoperiosteal flap was raised to expose the implant placement site. ANKYLOS Round Drill $\varnothing 3 \mathrm{~mm}$ with internal cooling was used to place initial osteotomy site so that it penetrates into the crestal bone. ANKYLOS Twist Drill, Ø $2 \mathrm{~mm}$ was used to get the depth of the implant to be placed. ANKYLOS Tri-Spade-Drill was skipped as the bone density was not good; instead Ankylos bone condensers with round cross-section of working tips were used for preparation of the implant site as the bone was very soft. Final site preparation was done using ANKYLOS conical reamer for the implant diameter to be used for widening the cavity to fit the conical core of the implants. Placement of endosseous Implants Ankylos ${ }^{\circledR} \mathrm{C}$ Implant was done in region of 11 and 21 was done, it resists a torque of $35 \mathrm{Ncm}$ indicating good primary stability.

Following a healing period of 3 months, periapical radiographs showed that oseointegration had been completed successfully therefore, no obstacle to moving onto the prosthesis stage. Healing screw or gingival former was placed using small incision without raising any flap followed by impression making after one week using ANKYLOS Balance C/ Repositioning Posts. (Fig. 3)

The trial metal porcelain crown was processed and finished to completion. An inter-occlusal registration was made. The occlusion of the crown was corrected. It was noticed that because of bone resorption the actual crown height was longer than the usual height so it was decided to correct the height using gingival porcelain and thus mimicking the extra height of the crown with adjacent gingival tissues. The final crowns were cemented onto the implants (Fig. 4). The patient was on 6-month follow up and after two years of followup, the crowns were found to be stable (Fig. 5) and the bone around the implants was found to be in adequate condition (Fig. 6). The patient was very positive of the completed restorations.

\section{Discussion}

Soft and hard tissue defects can be treated with surgical or prosthetic approaches. The original tissue contours can be mimicked with successful surgical treatment.[10,11] The disadvantages of surgical approach include need for bone augmentation, surgical costs, healing time, discomfort and unpredictability when large volume of tissue is missing. In such cases, prosthetic replacement is a more predictable approach for replacing the lost tissue architecture. The major advantage of these prostheses includes an aesthetically pleasing, functional restoration without undergoing any additional surgical procedures when a larger amount of tissue needs replacement. A clear understanding of the clinical requirements is essential before soft-tissue replacement with either fixed or removable prostheses is planned.[2]

Using this kind of approach has many advantages such as patient's comfort and self-confidence and cost effectiveness. Hence, its application is limited to situations where patient has got minimal visibility of his/her teeth during speech and smile and the desired aesthetic result is achievable.

\section{Conclusion}

Space evaluation in all three dimensions (mesio-distal, labio-lingual and apico-coronal) is importance with respect to the adjacent teeth and their periodontal ligaments. Design and timing of implant placement adds to the fourth dimension of implant planning. This clinical report has presented methods of using gingival colour ceramic materials to create cervical portion of the crown. As white component and pink component are equally important in dental aesthetics, we need to clearly understand the colour and form requirements before 
fabricating prosthesis. In cases of where as patient is not interested in bone augmentation or any other invasive surgical procedures this approach using gingival porcelain can be of great help while maintaining functional as well as esthetic demand of the patient without much intervention.

\section{References}

[1] Augthun M, Yildirim M, Spiekermann H, et al (1995): Healing of bone defects in combination with immediate implants using the membrane technique. Int J Oral Maxillofac Implants 10:421,

[2] Barzilay I, Tamblyn I. (2003) Gingival prostheses — A review. J Can Dent Assoc; 69:74-8.

[3] Buser D, BrLgger U, Lang NP, et al (1990): Regeneration and enlargement of jaw bone using guided tissue regeneration. Clin Oral Implant Res 1:22,

[4] Buser D, Dula K, Belser UC, et al (1995): Localized ridge augmentation using guided bone regeneration. II. Surgical procedure in the mandible. Int $\mathrm{J}$ Periodont Rest Dent 15:11,

[5] Buser D, Hirt HP, Dula K, et al (1992): GBR-technique/implant dentistry: Simultaneous application of barrier membranes around implants with periimplant bone defects. Schweiz Monatsschr Zahnmed 102:1491,

[6] Khoury F (1987) Die modifizierte Alveolar-Extensions-Plastik. Z Zahnlrzl Implant 01 3: 174

[7] Lustmann J, Lewinstein I (1995): Interpositional bone grafting technique to widen narrow maxillary ridge. Int J Oral Maxillofac Implants 10:568,

[8] Misch CM, Misch CE, Resnik RR, et al (1992): Reconstruction of maxillary alveolar defects with mandibular symphysis grafts for dental implants: A preliminary procedural report. Int J Oral Maxillofac Implants 7:360,

[9] Misch CM, Misch CE (1995): The repair of localized severe ridge defects for implant placement using mandibular bone grafts. Implant Dent 4:261:

[10] Oates T, Robinson M, Gunsolley (2003) J. Surgical therapies for the treatment of gingival recession. A systematic review. Ann Periodontol; 8:303-20.

[11] Rocuzzo M, Bunino M, Needleman I, (2002) periodontal plastic surgery for treatment of localized gingival recessions: A systematic review. J Clin Periodontol; 29:178-94.

[12] Rominger JW, Tripled RG (1994): The use of guided tissue regeneration to improve implant osseointegration. J Oral Maxillofac Surg 52:106,

[13] Tarnow D, Elian N, Fletcher P, et al. (2003) Vertical distance from the crest of bone to height of the interproximal papilla between adjacent implants. J Periodontol; 74:1785-788.

[14] Ten Bruggenkate CM, Kraaijenhagen HA, van der Kwast WAM, et al (1992): Autogenous maxillary bone grafts in conjunction with placement of I.T.I. endosseous implants: A preliminary report. Int J Oral Maxillofac Surg 21:81,

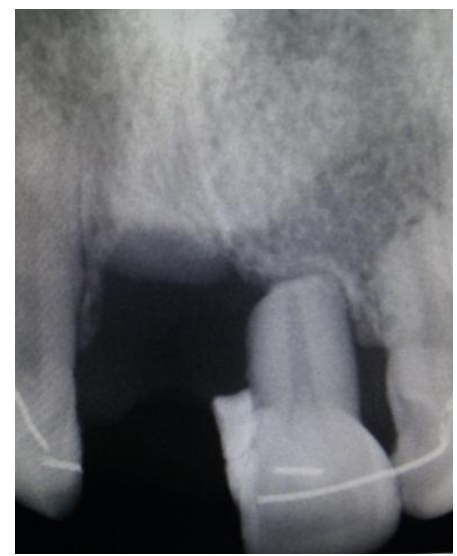

Figure 1 Pre Operative IOPA

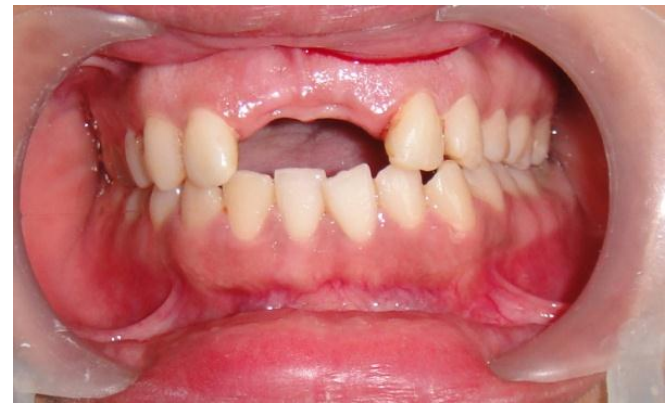

Figure 2 Pre Operative Picture 


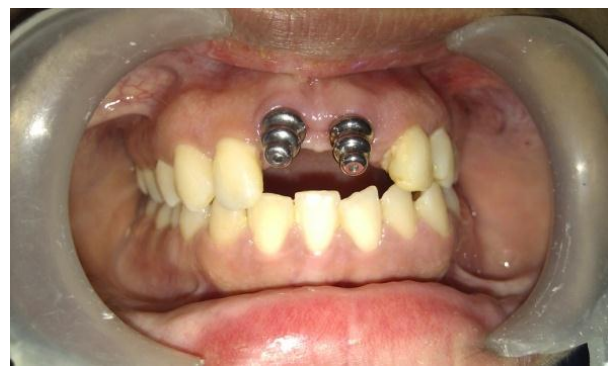

Figure 3 Picture showing Balance C/ Repositioning Posts

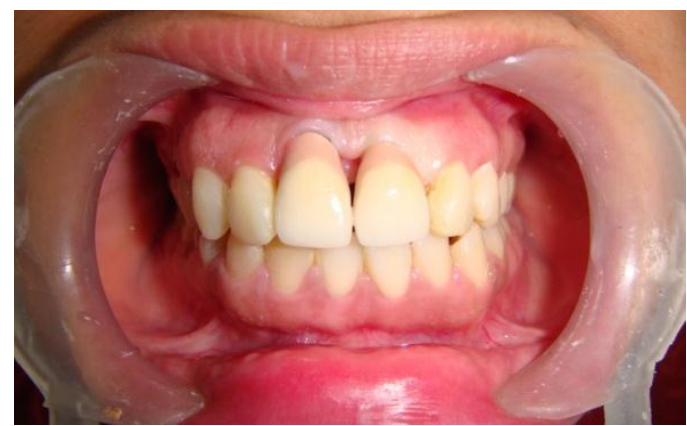

Figure 4 Post Operative just after Crown Cementation

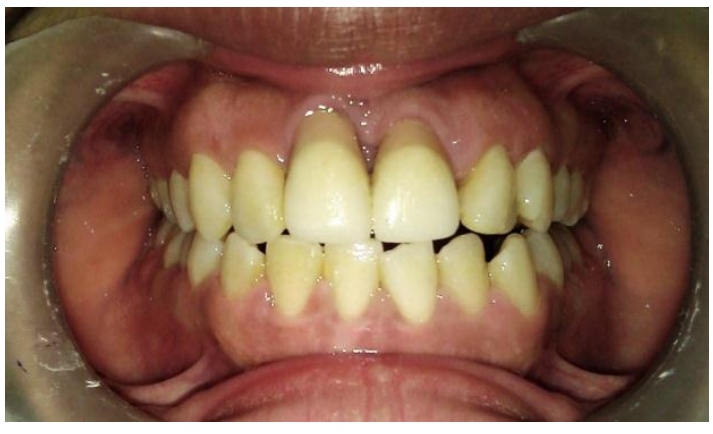

Figure 5 Post Operative 6 months after Crown Cementation

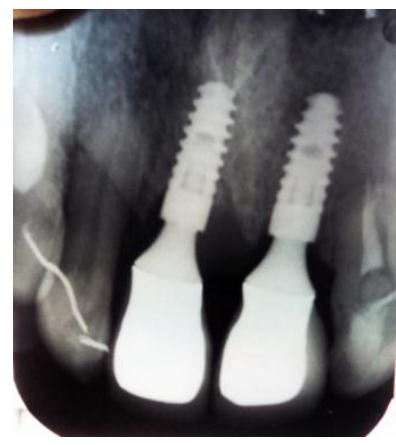

Figure 6 Post-Operative IOPA 


\section{Explanation to the Figures:}

Figure 1 Pre Op IOPA

Figure 2 Pre Op Picture

Figure 3 Picture showing Ankylos Balance C/ Repositioning Post

Figure 4 Post Operative just after Crown Cementation

Figure 5 Post Operative 6 months after Crown Cementation

Figure 6 Post-Operative IOPA 